\title{
Learning Bidding Strategies in Local Electricity Markets using a Nature-Inspired Algorithm
}

\author{
Fernando Lezama, Joao Soares, Ricardo Faia, Pedro Faria \\ GECAD, Polytechnic of Porto (ISEP/IPP), Portugal \\ Email: $\{f l z$, jan, rfmfa, pnf $\}$ isep.ipp.pt
}

\author{
Zita Vale \\ Polytechnic of Porto (ISEP/IPP), Portugal \\ Email: zav@isep.ipp.pt
}

\begin{abstract}
Local electricity markets (LEM) are a promising idea to foster the efficiency and use of renewable energy at the distribution level. However, how these local markets will be integrated into existing market structures, and to make the most profit from them, is still unclear. In this work, we propose a LEM framework based on bi-level optimization. In the upper level, end-users aim at maximizing profits, while the lower level represents the clearing market process. Moreover, a cascade integration to the wholesale market through an aggregator that acts after the LEM has been cleared is considered. Learning strategies using only available information can be a powerful tool to take the most advantage of LEM. To this end, we advocate the use of ant colony optimization (ACO), a nature-inspired technique, similar to that employed in machine learning. By using ACO, consumers, producers and prosumers, can learn the best strategies to maximize their profits without sharing private information and based solely on their experience.

Index Terms-Ant Colony Optimization; Evolutionary computation; Learning Strategy; Local energy market; Renewable energy.
\end{abstract}

\section{INTRODUCTION}

The recent investments of renewable generation at the distribution level are contributing to decentralization and decarbonization and motivating the proposition of new market models [1], [2]. In this scenario, local electricity markets (LEM) will provide a new framework for players to trade energy (most often of renewable type) and thus, contribute to lower carbon emissions. To fully realize the potential of LEMs, it is required sophisticated technology to be in place, namely smart grid communications and smart meter data [3], [4]. End-users can, therefore, be empowered to take a large part in the energy community and help to promote the transition to a sustainable energy system [1]. We believe that the competition fostered by LEMs, namely between the smart grid agents (or players) will allow local small producers and prosumers to participate and obtain more significant profits than the current policy allows, usually with feed-in tariff approaches. Also, local consumption will contribute to postpone grid investments and reduce grid losses increasing the overall operational efficiency [5].

Some works have proposed LEM structures using decentralized approaches and focusing on services such as flexibility

This work has received funding from the European Union's Horizon 2020 research and innovation programme under project DOMINOES (grant agreement No 771066), from FEDER Funds through COMPETE program and from National Funds through (FCT) under the projects UIDB/00760/2020, MASSociety (PTDC/EEI-EEE/28954/2017), and grants CEECIND/02887/2017, CEECIND/02814/2017, SFRH/BD/133086/2017. for distribution system operators (e.g., blockchain and peerto-peer trading as ICT). Research is also paying attention to the interactions between LEMs and central markets [6], [7]. The work in [8] presents a comprehensive market design and simulation of an LEM between 100 residential households using private blockchain technology to ensure data and energy trading without the need of a centralized entity. Authors in [9] propose peer-to-peer (P2P) trading with an exchange platform called STEP to integrate prosumers and make the interface between the wholesale market (WS) and prosumer communities. The work in [6] explains how the WS market and local flexibility market can be coupled to enable interaction between transmission and distribution. It discusses the possibility of prosumers as flexibility providers. The main stakeholders are the distribution system operator (DSO) and balance responsible parties that could be interested in buying this flexibility from aggregators and flexible devices. The work in [7] is a systematic review of the coordination schemes categorized as DSO leader, DSO follower, transmission system operator (TSO)-DSO interaction and independent LEMs without interaction with central electricity markets. According to this work, the coordination scheme that provides higher social welfare is the TSO-DSO interaction. However, such a platform is challenging to realize in practice since to optimal dispatch of all the resources TSO-DSO is computationally expensive.

In this work, we propose a LEM framework based on bilevel optimization and simulation. In the upper-level, end-users aim at maximizing profits, while the lower-level represents the clearing market process. Moreover, end-users have access to the wholesale market through an aggregator that acts after the LEM has been cleared. The aggregator can achieve profits for users and for itself by fixing a tariff based in the forecasted wholesale energy price. The bi-level problem can be optimally solved under the assumption that end-users share information regarding their bidding strategies. However, in practice, market players aim at maximizing their profits in a competitive manner and without exchange of private information. In such a scenario, learning strategies using only available information can be a powerful tool to take the most advantage of LEM. To this end, we advocate the use of ant colony optimization (ACO) [10], a nature-inspired technique, similar to that employed in machine learning, that has been applied successfully to different combinatorial problems and as a learning strategy in computer sciences. 
The contributions of the paper are:

- a bi-level optimization/simulation framework for bidding in LEM, including an aggregator coordination scheme for interoperability between LEM and wholesale market.

- a bidding learning framework based on ant colony optimization.

- an analysis of a case study based on realistic data to assess the impact of LEM participation, the use of ACO, and the impact of aggregator tariffs.

\section{LOCAL ELECTRICITY MARKET FRAMEWORK}

Since a local area cannot most likely maintain selfsufficiency, we assume that local end-users are able to trade energy with external sources. Therefore, energy should be procured from where it is produced at the cheapest cost to maintain an efficient system while considering losses, limitations, and energy needs. Figure 1 depicts the proposed framework, in which a LEM is considered allowing the trade of energy between end-users, but a cascading integration with the wholesale market is possible by means of an aggregator. For the proper functioning of the LEM, we consider three types of end-users, namely consumers, small producers (i.e., combined heat and power (CHP) generators), and prosumers (i.e., consumers with PV generation capabilities). Currently, prosumers with renewable generation capability are limited to self-consumed the produced energy and feed-in the excess to the grid at a fixed tariff. However, a market mechanism will turn end-users in active participants of the energy chain by allowing the exchange of energy between peers and fostering in that way the penetration of renewables. It is also assumed hierarchical cascading markets, where the initial trading is done locally (i.e., in the LEM), and further resources can be forwarded to larger WS markets. Different network tariffs might apply to accessing the local and external markets. We analyse in this paper different tariff imposed by the aggregator, to appreciate the effect of such tariff in local transactions and the use of the LEM. Also, social aspects (community trading) and the ability to consider local network congestion can be contributing factors to analyze in further work.

The LEM bidding problem is formulated as a bi-level optimization problem [11], [12]. The upper-level corresponds to the maximization of agents' profits, and the lower-level problem is the maximization of energy transacted in the LEM. Thus, the lower-level defines a clearing price depending on the bidding of agents modifying the profits that agents want to maximize in the upper-level.

Consider a set of consumer agents $I=1,2, \ldots N_{c}$, and producer agents $J=1,2, \ldots N_{p}$, where each agent $i$ wants to minimize its costs while agents $j$ want to maximize their profits. The optimization problem for each consumer agent (minimization of costs) can be formulated as:

$$
\operatorname{minimize} \quad C_{i}=\sum_{j} c p \cdot x_{j, i}+c^{A G G} \cdot E_{b u y_{g r i d}, i}
$$

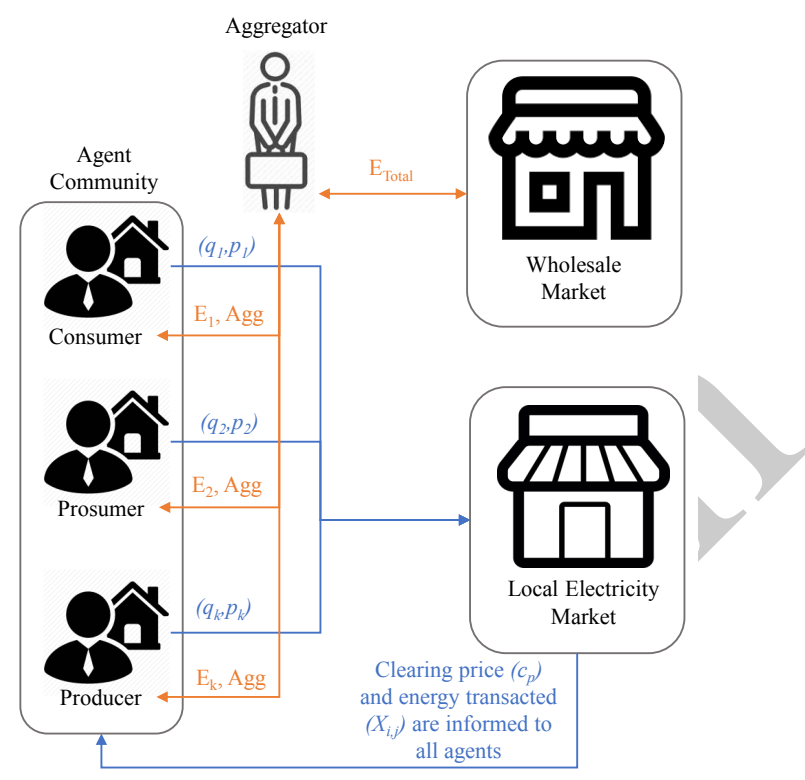

Fig. 1. Considered LEM and the integration with the wholesale market.

where $c p$ is the LEM clearing price, $x_{j, i}$ contains the energy sold by agent $j$ to agent $i$ in the LEM, $c^{A G G}$ is the aggregator tariff, and $E b u y_{i, A G G}$ is the energy bought by agent $i$ from the aggregator.

On the other hand, producer agents try to maximize their profits as follows:

$\operatorname{maximize} \quad P_{j}=\sum_{i} c p \cdot x_{j, i}+c^{F} \cdot$ Esell $_{j, g r i d}-c^{m} * G_{j}$

where $c p$ is the LEM clearing price (equal for buyers and sellers), $x_{j, i}$ contains the energy sold by agent $j$ to agent $i, c^{F}$ is the feed-in tariff, Esell j,grid $_{\text {id }}$ is the energy sold by agent $j$ to the grid, and $c^{m} * G_{j}$ represents the marginal cost associated to $j$. We assume that $c^{m}=0$ for PV generation, and $c^{m}=c_{C H P}^{m}\left(G_{j}\right)$ (i.e., the marginal cost associated to a CHP generator) is defined as a monotone decreasing function [11]:

$$
c_{C H P}^{m}\left(G_{j}\right)=\frac{b_{C H P} \cdot \sqrt{G_{j}}}{G_{j}}
$$

where $b_{C H P}$ is a cost factor of the CHP generation unit and $G_{j}$ is the energy produced by the CHP. We also assume that producer agents with CHP capability cannot sell energy to the grid at the feed-in tariff, and can also participate in the LEM. On the other hand, prosumers used the PV for selfconsumption first, while the excess of energy can be sold either in the LEM or injected to the grid at the feed-in tariff.

The lower-level problem is modeled as an symmetric pool market, in which bids and offers are allocated using a merit order procedure to determine the supply and demand curves [13]. Thus, the LEM clearing price is determined as the price in which demand equals supply. 
In our simulations, we are interested in increasing the overall mean profits of the system and at the same time provide solutions that distribute the earnings among all agents. We measure this with an objective function modeled as:

$$
\max \sum_{j}\left(P_{j}\right) / N_{p}-\sum_{i}\left(C_{i}\right) / N c
$$

where $P_{j}$ and $C_{i}$ are objectives in conflict since agents want to achieve the best result for their own. A detailed mathematical formulation of the problem can be found in [11], [12], including a detailed description of the decision variables and restrictions of the problem.

\section{Ant COLONy Optimization}

Ant Colony Optimization (ACO) is a nature-inspired problem-solving approach that simulates the social behaviour of ants depositing pheromone on the ground to mark favourable paths to food. Such paths are reinforced by other members of the colony depending on their quality. The first ACO algorithm was called as ant system (AS) [14], and since then, many variants (such as the MAX-MIN Ant System [15] or the Ant Colony System [16]) have been applied to diverse optimization problems. We adopt the implementation of [17] in which a simple ACO algorithm was used to learn optimal bidding policies from a discrete set of bidding options, and for each independent agent. Such implementation is compared to those techniques of machine learning, and can be employed in a distributed way to avoid the issue of information sharing between players. The pseudocode of the simple ACO algorithm is presented in 1 , and the explanation of each phase is explained in the following subsections.

\begin{tabular}{l}
\hline Algorithm 1 ACO pseudocode \\
\hline 1: Set parameters, initialize pheromone trails. \\
2: while termination condition not met do \\
3: Construct Ant Solutions. \\
4: $\quad$ Evaluate fitness of ants. \\
5: Apply Local Search (optional). \\
6: Update Pheromones. \\
7: end while \\
\hline
\end{tabular}

\section{A. Solution construction phase}

A solution containing price and quantity to bid in the LEM for each agent $K=\left\{1,2, \ldots N_{k}\right\}$, where $N_{k}$ is the number of agents, can be represented through a vector $\vec{x}=\left\{\left[q_{k, t}\right] \cup\left[p_{k, t}\right]\right\}$ including the bids for quantity and price that the $k$ th agent will send to the LEM at each time $t$. We use a sign convention in which a positive quantity represents a bid (i.e., buying in the market), while a negative quantity represents an offer (i.e., selling in the LEM).

Consumers can put a bid of quantity within allowed bounds $\left[0, L_{\max }\right]$ (i.e., between 0 and their maximum consumption), while producer can offer within the bounds $\left[-P_{\max }, 0\right]$ (i.e., between 0 and their maximum production capacity). The price bounds for bids/offers are within the range $\left[c^{F}, c^{A g g}\right]$. Such bounds are discretized $L$ equal parts ( $L$ is assumed to be a discretization level). Therefore, the continues variables $q_{i}$ and $p_{i}$ are mapped to a discrete space $q_{(i, l)}=\left\{q_{(i, 1)}, q_{(i, 2)}, \ldots, q_{(i, L)}\right\}$ and $p_{(i, l)}=\left\{p_{(i, 1)}, p_{(i, 2)}, \ldots, p_{(i, L)}\right\}$, to turn the problem into a combinatorial one that can be solved by ACO.

A set of $m$ artificial ants constructs solutions from elements of a finite set of available solution components $C=\left\{q_{i}, p_{j}\right\}$, $i=1, \ldots, T, j=1, \ldots T$. A solution construction starts from an empty partial solution $s^{p}=\emptyset$. At each construction step, the partial solution $s^{p}$ is extended by adding a feasible solution component $\mathbf{N}\left(s^{p}\right) \subseteq \mathbf{C}$ (i.e., a bid/offer of quantity and price from the available discrete set). The selection of a solution component is guided by a stochastic mechanism biased by a pheromone associated with each element $\mathbf{N}\left(s^{p}\right)$ :

$$
\begin{aligned}
\operatorname{Prob}_{q(i, l)} & =\frac{\tau_{q(i, l)}^{\alpha} \cdot \eta_{q(i, l)}^{\beta}}{\sum_{c_{q_{i}} \in \mathbf{N}\left(s^{p}\right)} \tau_{q(i, l)}^{\alpha} \cdot \eta_{q(i, l)}^{\beta}} \\
\operatorname{Prob}_{p(i, l)} & =\frac{\tau_{p(i, l)}^{\alpha} \cdot \eta_{p(i, l)}^{\beta}}{\sum_{c_{p_{i}} \in \mathbf{N}\left(s^{p}\right)} \tau_{p(i, l)}^{\alpha} \cdot \eta_{p(i, l)}^{\beta}}
\end{aligned}
$$

where the parameters $\alpha$ and $\beta$ control the relative importance of the pheromone or probability (i.e., $\tau$ ) versus the heuristic information (i.e., $\eta$ ). Pheromone and heuristic matrices used for the bidding problem are defined in the next subsection.

\section{B. Pheromone and heuristic information}

A pheromone value is associated with each potential assignment of a value to a variable. In this work, the pheromone trail is modelled as two matrices $\tau_{q}$ and $\tau_{p}$, both of dimension $T \times L$, where $T$ is the number of periods, and $L$ is the discretization level. The pheromone trail stores information learned from the ants and indicates the bid/offer of quantity and price that the agent put in the LEM for each period. The information is reinforced according to the fitness of each ant after obtaining a complete solution. The better the fitness, the more the reinforcement of matrices $\tau_{q}$ and $\tau_{p}$.

On the other hand, the heuristic information matrices $\eta_{q}$ and $\eta_{p}$, also both of dimension $T \times L$, are defined with information related to the decision of quantity as:

$$
\eta_{q(i, l)}= \begin{cases}q_{i, l} & \text { if } q_{i, l} \geq 0 \quad \text { (for consumers) } \\ \left|q_{i, L-l+1}\right| & \text { otherwise (for producers) }\end{cases}
$$

where $q_{i, l}$ is the discrete bid/offer value of energy quantity. When $q_{i, l} \geq 0$, the matrix reinforce the decision of buying as much as possible in the local market. On the contrary, when $q_{i, l}<0$ (representing a producer), the matrix is flip to favour major quantities to sell into the LEM.

A similar differentiation is done for the heuristic information of price:

$$
\eta_{p(i, l)}= \begin{cases}1 / p_{i, l} & \text { if } q_{i, l} \geq 0 \quad \text { (for consumers) } \\ p_{i, l} & \text { otherwise (for producers) }\end{cases}
$$




\section{Update of pheromone trails}

At each iteration, the pheromone trail is updated to increase the values associated with promising solutions, and to decrease those that are associated with bad ones. After the construction of all the solutions, the pheromone trails are modified through and evaporation and reinforcement as:

$$
\begin{aligned}
\tau_{q(i, l)} & \leftarrow(1-\rho) \cdot \tau_{q(i, l)}+\sum_{m=1}^{M} \Delta \tau_{q(i, l)}^{m} \\
\tau_{p(i, l)} & \leftarrow(1-\rho) \cdot \tau_{p(i, l)}+\sum_{m=1}^{M} \Delta \tau_{p(i, l)}^{m}
\end{aligned}
$$

where $\rho$ is an evaporation rate parameter defined by the user to simulate the evaporation process of pheromone, and $\Delta \tau_{p(i, l)}$ and $\Delta \tau_{q(i, l)}$ are the pheromone contribution of ant $m$ to the trace calculated as:

$$
\begin{aligned}
& \Delta \tau_{q(i, l)}^{m}= \begin{cases}1 / F_{i} t_{m} & \text { if } q_{i, l} \in \text { sol }^{m} \wedge F_{i t_{m}} \geq 0 \\
\mid \text { Fit }_{m} \mid & \text { if } q_{i, l} \in \text { sol }^{m} \wedge \text { Fit }_{m}<0 \\
0 & \text { otherwise }\end{cases} \\
& \Delta \tau_{p(i, l)}^{m}= \begin{cases}1 / \text { Fit }_{m} & \text { if } p_{i, l} \in \text { sol }^{m} \wedge F i t_{m} \geq 0 \\
\left|F i t_{m}\right| & \text { if } p_{i, l} \in \text { sol }^{m} \wedge F i t_{m}<0 \\
0 & \text { otherwise }\end{cases}
\end{aligned}
$$

where $F i t_{m}$ is the fitness of the solution constructed by ant $m$ (denoted by sol $^{m}$ ). The inverse and absolute value are used because the fitness is associated with the costs/profits of each agent, which in this applications can take negative values (representing profits). This procedure reinforces the traces of the best solutions found. The iterative process is repeated until a termination condition is met, such as the number of generations.

\section{Distributed ACO for each agent}

The reader can consult [17] for details on the tuning of parameters and implementation of the used ACO. It is worth to notice that agents searches for the optimal bidding in the LEM. Therefore, the ACO is adapted to be applied to each agent independently, providing them with their own pheromone matrix, and allowing distributed learning without sharing information. The parameters used in this study are those as the best set determined in [17] and presented in Table I. A comparison of the performance of such a technique with other nature-inspired algorithms is left as further research, but the reader can consult [12], [18] in this regard.

\section{CAse Study}

We adopt a case study with nine agents, in which 3 of them are consumers, 3 are prosumers (i.e., consumers with PV generation), and 3 are small CHP producers [12]. Sample power profiles of residential houses and PV systems are built using the open datasets available at PES ISS website ${ }^{1}$. A randomized function with a uniform distribution, $20 \%$ around

\footnotetext{
${ }^{1}$ Open data online at http://sites.ieee.org/pes-iss/data-sets/
}

TABLE I

EXP1 DEFAULT AND TESTED VALUES FOR ACO PARAMETER TUNING.

\begin{tabular}{l|ll} 
Parameter & Tested & Recommendation \\
\hline$\rho$ & {$[0.1,0.3,0.5,0.7,0.9]$} & 0.3 \\
$\alpha$ & {$[0.5,1,2,3,4]$} & 0.5 \\
$\beta$ & {$[0.5,1,2,3,4]$} & 0.5 \\
$m$ & {$[5,10,20,30,40,50]$} & 20 \\
Iter & {$[100,200,300,400,500,1000]$} & 500 \\
\hline \multicolumn{2}{l}{ An empirical study was performed to determine these values }
\end{tabular}

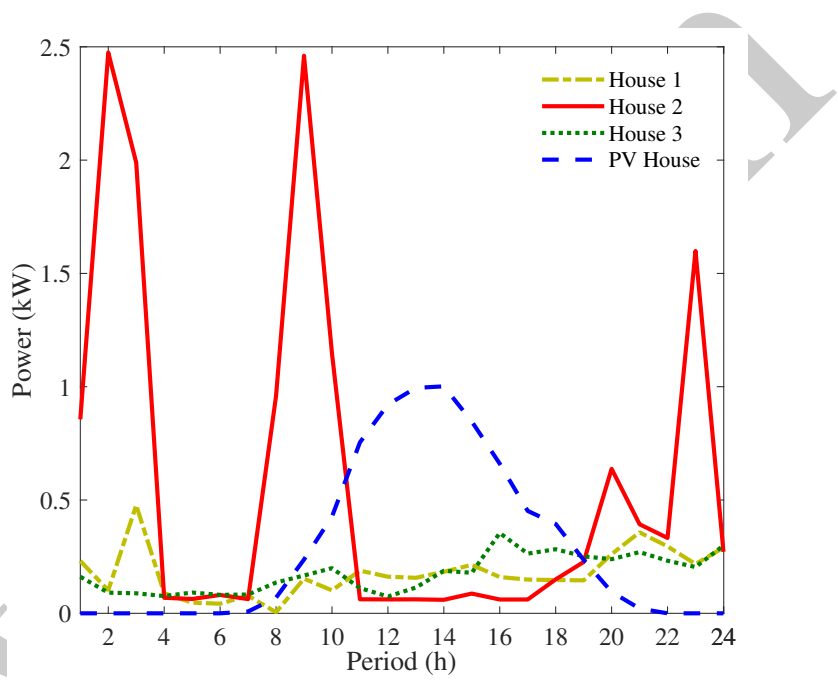

Fig. 2. Profiles used in the case study. Ranges of power (in $\mathrm{kW}$ ): house 1 [0.18-0.48], house 2 [0.06-2.50], house 3 [0.07-0.36], PV (house) [0-1].

the standard profiles showed in Figure 2, is used to generated data for the case study. Figure 2 also provides the power ranges of the base profiles. Agents eqquiped with CHPs generators have a maximum generation capacity of $2 \mathrm{~kW}$ and a marginal cost calculated with Eq. (3) with a factor $b_{C H P}=0.10$ EUR $/ \mathrm{kWh}$. The feed-in tariff has been set to $c^{F}=0.095$ EUR/kWh following a Portuguese scenario, while the aggregator tariff $c^{A G G}$ has been varied and from 0.10 to 0.25 $\mathrm{EUR} / \mathrm{kWh}$ plus the wholesale market price (which in average is $0.05 \mathrm{EUR} / \mathrm{kWh}$ ). We assume that the aggregator tariff include network fees (like for instance taxes, transportation, distribution [19]) and a margin of profit for itself.

\section{REsults}

In this section, we present the results of the simulations. The experiments are done to analyze the impact of LEM and the effect on the bounds of price imposed by the aggregator. For this reason, we run the simulations considering a variation on the price of the aggregator from $0.10 \mathrm{EUR} / \mathrm{kWh}$ to 0.25 EUR/kWh. Fig. 3 shows the energy transacted in the LEM and sold by the aggregator (AGG) in the different fee variations scenarios. A visible trend is captured here and depicted in the figure. The higher the price imposed by the AGG, the higher the energy transacted in the LEM. This happens because the consumers will avoid higher fees with the aggregator and try to decrease their costs by negotiating energy in the LEM. This, in turn, causes the LEM clearing prices to increase because 
TABLE II

COMPARISON OF RESULTS IN THE CONSIDERED FEE VARIATIONS WITH AND WITHOUT LEM (EUR)

\begin{tabular}{|c|c|c|c|c|c|c|c|c|c|c|}
\hline & \multicolumn{3}{|c|}{ Consumers } & \multicolumn{3}{|c|}{ Prosumers } & \multicolumn{3}{|c|}{ Producers } & \multirow{2}{*}{ Total } \\
\hline & $\mathbf{A 1}$ & A2 & $\mathbf{A 3}$ & A4 & A5 & A6 & A7 & A8 & A9 & \\
\hline No LEM-AGG10 & 0.64 & 2.02 & 0.61 & -0.17 & 1.35 & -0.16 & 0.00 & 0.00 & 0.00 & 4.29 \\
\hline LEM+AGG10 & 0.53 & 1.71 & 0.50 & -0.24 & 1.07 & -0.22 & -0.09 & 0.03 & -0.10 & 3.19 \\
\hline No LEM-AGG15 & 0.86 & 2.70 & 0.81 & -0.04 & 1.97 & -0.07 & 0.00 & 0.00 & 0.00 & 6.23 \\
\hline LEM+AGG15 & 0.62 & 2.07 & 0.61 & -0.14 & 1.49 & -0.17 & -0.12 & -0.26 & -0.14 & 3.95 \\
\hline No LEM-AGG20 & 1.07 & 3.38 & 1.01 & 0.08 & 2.59 & 0.03 & 0.00 & 0.00 & 0.00 & 8.16 \\
\hline LEM+AGG20 & 0.82 & 2.50 & 0.70 & -0.04 & 1.66 & -0.04 & -0.27 & -0.24 & -0.35 & 4.74 \\
\hline No LEM-AGG25 & 1.29 & 4.06 & 1.22 & 0.21 & 3.20 & 0.12 & 0.00 & 0.00 & 0.00 & 10.10 \\
\hline LEM+AGG25 & 0.89 & 2.82 & 0.78 & 0.09 & 2.00 & -0.03 & -0.23 & -0.25 & -0.81 & 5.26 \\
\hline
\end{tabular}

*Negative values represent profits

the demand also increases (consumers shift from aggregator to LEM, cf. Fig. 4).

Table II depicts the comparison of results in the considered AGG fee variations with and without LEM. The costs/profits are shown by a group of consumers, prosumers, and producers (agent A1 to A9). The last column (total) represents the total amount in EUR, i.e., the total cost of the system, where the lower is the best case. It can be seen that the use of LEM drastically reduces the cost of the system by $26-48 \%$ (cf. AGG 25 case). This trend is more noticeable when the AGG fee increases and the producers (A7 to A9) obtain more gains by selling energy to the LEM, instead of adopting the feedin tariff or selling at a loss (cf. LEM+AGG10, A8). In this particular case simulation, the LEM cannot outweigh the fee variations experimented with the AGG. However, in a realistic scenario, we believe these variations should be reasonably less. The LEM could eventually outweigh more AGG fee variations (which also reflects the fluctuations in the wholesale electricity market) if more producers were available to offer energy. Fig. 4 depicts the clearing prices (CP) in the four scenarios of AGG fee variations, including the fixed feed-in tariff and the 4 AGG fee variations. This figure confirms that the LEM model is working correctly, namely having lower CP

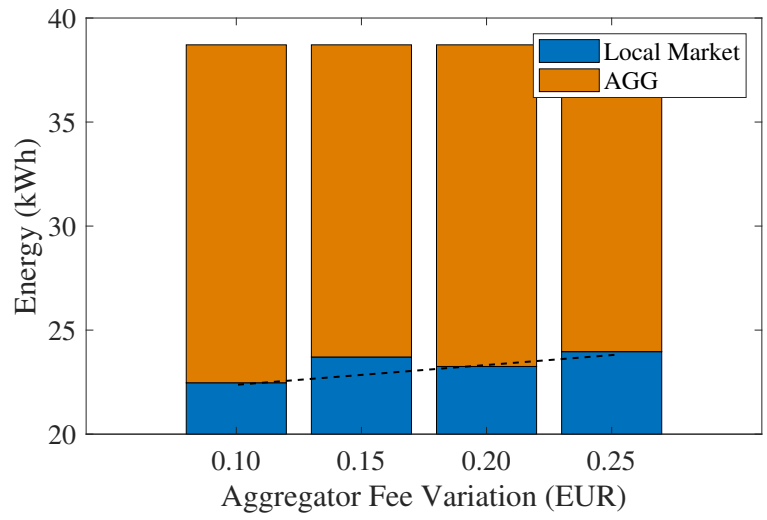

Fig. 3. Energy transacted in LEM and sold by the aggregator (AGG) in the different fee variations scenarios. prices when there is low demand from the consumers or high renewable generation from the producers. Both cases produce the lowest clearing prices in LEM. LEMs can highly contribute to improve profits for small renewables and distributed energy sources (DERs) at the distribution level and, at the same time, reduce the energy costs for consumers. The social welfare of introducing DERs at the local level instead of larger utility-scale centralized systems is therefore increased with the opportunity of LEMs. Still, their benefits deserve more studies depending on each location and country.

\section{CONCLUSIONS}

LEMs can unlock several benefits for end-users, utilities, and the system as a whole. Nonetheless, due to the interactions of different actors following diverse objectives, emerging issues need to be studied to take full advantage of energy traded at the local level. Moreover, the uncertainty of renewable penetration and independent users acting in such an environment pose a new challenge into a problem already complex. In this paper, a simulation framework for end-users acting in a LEM, and the wholesale market through an aggregator, was proposed. We employed a nature-inspired algorithm, the ACO system, for learning bidding strategies without sharing private information. The impact of aggregator tariffs was analyzed, showing that LEM improves end-users' profits despite the aggregator tariff. It was also demonstrated that the LEM clearing price tends to be low in periods with low demand or excess in renewable generation, showing that consumers are favored under the case study and proposed framework. In contrast, generators tend to reduce their possible profits. Despite the observed benefits, several assumptions were made in this study, so different research lines can be followed. For instance, the effect of offer and demand under various case studies within this framework is a logical step to take. Also, incorporating longer time horizons and other markets (such as intra-day markets) is worth exploring. Finally, network constraints, the uncertainty of renewables, and the participation of a distribution network operator (DSO) to validate the transactions in the LEM should be taken into account in future studies. 


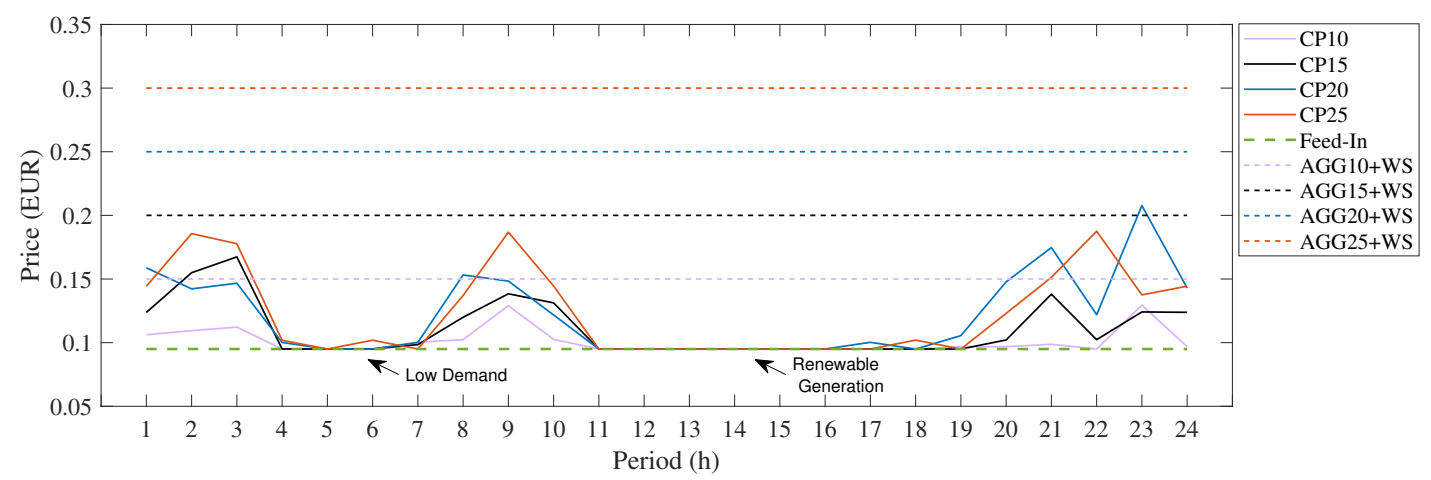

Fig. 4. Market clearing prices in LEM in the different AGG fee variations compared with the feed-in and AGG prices.

\section{REFERENCES}

[1] F. Lezama, J. Soares, P. Hernandez-Leal, M. Kaisers, T. Pinto, and Z. Vale, "Local energy markets: Paving the path towards fully transactive energy systems," IEEE Transactions on Power Systems, 2018.

[2] Í. Munné-Collado, E. Bullich-Massagué, M. Aragüés-Peñalba, and P. Olivella-Rosell, "Local and micro power markets," Micro and Local Power Markets, pp. 37-96, 2019.

[3] S. Chakraborty, P. Hernandez-Leal, and M. Kaisers, "Coordinating distributed and flexible resources: A case-study of residential cooperatives," in 2018 IEEE PES Innovative Smart Grid Technologies Conference Europe (ISGT-Europe). IEEE, 2018, pp. 1-6.

[4] J. Soares, T. Pinto, F. Lezama, and H. Morais, "Survey on complex optimization and simulation for the new power systems paradigm," Complexity, vol. 2018, 2018.

[5] P. Olivella-Rosell, E. Bullich-Massagué, M. Aragüés-Peñalba, A. Sumper, S. Ø. Ottesen, J.-A. Vidal-Clos, and R. VillafáfilaRobles, "Optimization problem for meeting distribution system operator requests in local flexibility markets with distributed energy resources," Applied energy, vol. 210, pp. 881-895, 2018.

[6] P. Olivella-Rosell, S. S. Torbaghan, and M. Gibescu, "Coupled local power markets," Micro and Local Power Markets, p. 165-191, 2019.

[7] Y. Tohidi, M. Farrokhseresht, and M. Gibescu, "A review on coordination schemes between local and central electricity markets," in 2018 15th International Conference on the European Energy Market (EEM). IEEE, 2018, pp. 1-5.

[8] E. Mengelkamp, B. Notheisen, C. Beer, D. Dauer, and C. Weinhardt, "A blockchain-based smart grid: towards sustainable local energy markets," Computer Science-Research and Development, vol. 33, no. 1-2, pp. 207214,2018

[9] J. M. Zepter, A. Lüth, P. C. del Granado, and R. Egging, "Prosumer integration in wholesale electricity markets: Synergies of peer-to-peer trade and residential storage," Energy and Buildings, vol. 184, pp. 163$176,2019$.

[10] M. Dorigo, M. Birattari, and T. Stutzle, "Ant colony optimization," IEEE computational intelligence magazine, vol. 1, no. 4, pp. 28-39, 2006.

[11] E. M. Mengelkamp, "Engineering local electricity markets for residential communities," Ph.D. dissertation, Karlsruher Institut für Technologie (KIT), 2019, 37.06.01; LK 01.

[12] F. Lezama, J. Soares, and Z. Vale, "Optimal bidding in local energy markets using evolutionary computation," in International Conference on Intelligent System Application to Power Systems (ISAP), July 2019, pp. 1-8.

[13] S. Parsons, M. Marcinkiewicz, J. Niu, and S. Phelps, Everything you wanted to know about double auctions, but were afraid to (bid or) ask. Department of Computer \& Information Science, University of New York, 2006.

[14] M. Dorigo, V. Maniezzo, and A. Colorni, "Ant system: optimization by a colony of cooperating agents," IEEE Transactions on Systems, Man, and Cybernetics, Part B (Cybernetics), vol. 26, no. 1, pp. 29-41, 1996.

[15] T. Stützle and H. H. Hoos, "Max-min ant system," Future generation computer systems, vol. 16, no. 8, pp. 889-914, 2000.
[16] M. Dorigo and L. M. Gambardella, "Ant colony system: a cooperative learning approach to the traveling salesman problem," IEEE Transactions on evolutionary computation, vol. 1, no. 1, pp. 53-66, 1997.

[17] F. R. S. J. F. P. Lezama, F. and Z. Vale, "Learning bidding strategies in local electricity markets using ant colony optimization," in IEEE Congress on Evolutionary Computation (CEC). IEEE, 2020, pp. 22-35.

[18] J. Soares, F. Lezama, B. Canizes, and Z. Vale, "Wcci/gecco 2020 competition evolutionary computation in uncertain environments: A smart grid application," 2019.

[19] S. Burger, J. P. Chaves-Ávila, C. Batlle, and I. J. Pérez-Arriaga, "A review of the value of aggregators in electricity systems," Renewable and Sustainable Energy Reviews, vol. 77, pp. 395 - 405, 2017. [Online]. Available: http://www.sciencedirect.com/science/article/pii/S1364032117305191 